Discussiones Mathematicae

Differential Inclusions, Control and Optimization 33 (2013) 205-219

doi:10.7151/dmdico.1151

\title{
ON PERIODIC OSCILLATIONS FOR A CLASS OF FEEDBACK CONTROL SYSTEMS IN HILBERT SPACES
}

\author{
NGUYen VAn Loi \\ Faculty of Fundamental Science \\ PetroVietNam University, Viet Nam \\ e-mail: loinv@pvu.edu.vn
}

\begin{abstract}
In this paper, by using the topological degree theory for multivalued maps and the method of guiding functions in Hilbert spaces we deal with the existence of periodic oscillations for a class of feedback control systems in Hilbert spaces.
\end{abstract}

Keywords: semilinear differential inclusion, periodic solution, guiding function.

2010 Mathematics Subject Classification: 34A60, 34H05, 34C25.

\section{INTRODUCTION}

The existence of periodic solutions for semilinear differential inclusions in Banach spaces was studied by a number of researchers (see, e.g., [12] and the references therein). The usual way for the investigation of this problem is to apply the method of integral multivalued operators or the method of the translation multivalued operator.

In the present paper, by combining the topological method and the method of guiding functions in Hilbert spaces (see $[13,14,16]$ ) we study the periodic oscillations in control systems governed by semilinear differential inclusions in Hilbert spaces. In comparison with the previous investigations, we consider the control function subject to a differential inclusion whose right-hand side depends on the state function, i.e., we consider the feedback control problem.

The paper is organized in the following way. In the next section we recall some basic facts from the theory of multivalued maps and the theory of linear Fredholm operators. The statement of the problem and the main result are given in Section 3. 


\section{PRELIMINARIES}

\subsection{Multimaps}

Let $\left(\mathcal{X}, d_{\mathcal{X}}\right)$ and $\left(\mathcal{Z}, d_{\mathcal{Z}}\right)$ be metric spaces. By the symbols $P(\mathcal{Z})[K(\mathcal{Z})]$, we denote the collections of all nonempty [resp., nonempty compact] subsets of $\mathcal{Z}$. If $\mathcal{Z}$ is a normed space, the symbols $K v(\mathcal{Z}), C v(\mathcal{Z})$ denote the collections of nonempty compact convex and nonempty convex closed subsets of $\mathcal{Z}$, respectively.

A multivalued map (multimap) $\Sigma: \mathcal{X} \rightarrow P(\mathcal{Z})$ is said to be upper semicontinuous (u.s.c.) if for every open set $V \subset \mathcal{Z}$, the set $\Sigma_{+}^{-1}(V)=\{x \in \mathcal{X}: \Sigma(x) \subset V\}$ is open in $\mathcal{X}$.

An u.s.c. multimap $\Sigma: \mathcal{X} \rightarrow K(\mathcal{Z})$ is said to be completely u.s.c. if its restriction on each bounded subset $\Omega \subset \mathcal{X}$ is compact, i.e., the set $\Sigma(\Omega)$ is relatively compact in $\mathcal{Z}$.

Definition 1 (see, e.g., $[3,4,8,9,15])$. A set $\mathcal{M} \in K(\mathcal{Z})$ is said to be aspheric (or $U V^{\infty}$, or $\infty$-proximally connected) if for every $\varepsilon>0$ there exists $\delta, 0<\delta<\varepsilon$, such that for each $n=0,1,2, \ldots$ every continuous map $g$ : $S^{n} \rightarrow O_{\delta}(\mathcal{M})$ can be extended to a continuous map $\widetilde{g}: B^{n+1} \rightarrow O_{\varepsilon}(\mathcal{M})$, where $S^{n}=\left\{x \in \mathbb{R}^{n+1}:\|x\|=1\right\}$ and $B^{n+1}=\left\{x \in \mathbb{R}^{n+1}:\|x\| \leq 1\right\}$.

Definition 2 (see [11]). A nonempty compact space $\mathcal{A}$ is said to be an $R_{\delta}$-set if it can be represented as the intersection of a decreasing sequence of compact, contractible spaces.

Definition 3 (see [8]). An u.s.c. multimap $\Sigma: \mathcal{X} \rightarrow K(\mathcal{Z})$ is said to be a $J$-multimap $(\Sigma \in J(\mathcal{X}, \mathcal{Z}))$ if every value $\Sigma(x), x \in \mathcal{X}$ is an aspheric set.

Let us recall (see, e.g., $[5,8])$ that a metric space $\mathcal{X}$ is called the absolute neighborhood retract (the ANR-space) if for each homeomorphism $h$ taking it onto a closed subset of a metric space $\mathcal{X}^{\prime}$, the set $h(\mathcal{X})$ is a retract of its open neighborhood in $\mathcal{X}^{\prime}$. If the set $h(\mathcal{X})$ is a retract of the whole space $\mathcal{X}^{\prime}$, it is called the absolute retract (the AR-space).

Notice that the collection of the ANR-spaces is broad enough. In fact, the following assertion holds true (see [5]).

Proposition 4. A compact subset of a finite-dimensional space is the ANR-space if and only if it is locally contractible.

Remark 5. By the known Whitney embedding theorem, the previous proposition implies that each compact finite-dimensional manifold is the ANR-space.

Furthermore, the union of a finite number of convex closed subsets in a normed space is the ANR-space. 
ON PERIODIC OSCILLATIONS FOR A CLASS OF ...

Proposition 6 (see [8]). Let $\mathcal{Z}$ be an $A N R$-space. In each of the following cases a u.s.c. multimap $\Sigma: \mathcal{X} \rightarrow K(\mathcal{Z})$ is a J-multimap: for each $x \in \mathcal{X}$ the value $\Sigma(x)$ is

(a1) a convex set;

(a1) a contractible set;

(a3) an $R_{\delta}$-set;

(a4) an AR-space.

In particular, every continuous map $\sigma: \mathcal{X} \rightarrow \mathcal{Z}$ is a J-multimap.

Definition 7. By $J^{c}(\mathcal{X}, \mathcal{Z})$ we will denote the collection of all multimaps $G: \mathcal{X} \rightarrow$ $K(\mathcal{Z})$ of the form $G=\Sigma_{q} \circ \cdots \circ \Sigma_{1}, q \geq 1$, where $\Sigma_{i} \in J\left(\mathcal{X}_{i-1}, \mathcal{X}_{i}\right), i=1,2, \ldots, q$, $\mathcal{X}_{0}=\mathcal{X}, \mathcal{X}_{q}=\mathcal{Z}$ and $\mathcal{X}_{i}$ for $1<i<q$ are open subsets of normed spaces.

Let us recall (see, e.g., [1]) that if $U$ is an open bounded subset of a Banach space $E$ and $F: \bar{U} \rightarrow K(E)$ is a compact $J^{c}$-multimap such that $x \notin F(x)$ for all $x \in \partial U$, then for the corresponding multifield $i-F$, where $i$ denotes the inclusion map, the topological degree $\operatorname{deg}(i-F, \bar{U})$ is well-defined and has all usual properties.

\subsection{Fredholm operators}

Definition 8 (see, e.g., [7]). A linear operator $L: \operatorname{dom} L \subseteq X \rightarrow Y$ is called Fredholm of index zero if

(1) $\operatorname{ImL}$ is closed in $Y$;

(2) KerL and Coker $L$ have the finite dimension and

$$
\operatorname{dimKerL}=\operatorname{dimCoker} L .
$$

For Banach spaces $X$ and $Y$ let us denote by $\mathcal{L}(X, Y)$ the set of all linear bounded operators from $X$ to $Y$. If $Y$ coincides with $X$, then instead of $\mathcal{L}(X, Y)$ we use the notation $\mathcal{L}(X)$.

Throughout this paper by $H$ we denote a separable Hilbert space which is compactly embedded in a separable Banach space $E$ with the relation of norms

$$
\|w\|_{E} \leq q\|w\|_{H}, \quad \forall w \in H
$$

where $q>0$. Let $\left\{e_{n}\right\}_{n=1}^{\infty}$ be an orthonormal basis of $H$. For every $n \in \mathbb{N}$, let $H_{n}$ be an $n$-dimensional subspace of $H$ with the basis $\left\{e_{k}\right\}_{k=1}^{n}$ and $P_{n}$ be a projection of $H$ onto $H_{n}$. By $\langle\cdot, \cdot\rangle_{H}$ we denote the inner product in $H$. In the sequel everywhere the symbol $I$ denotes the interval $[0, T]$. By $C(I, H)\left[L^{2}(I, H)\right]$ 
we denote the spaces of all continuous [respectively, square summable] functions $u: I \rightarrow H$ with usual norms

$$
\|u\|_{C}=\max _{t \in I}\|u(t)\|_{H} \quad \text { and } \quad\|u\|_{2}=\left(\int_{0}^{T}\|u(t)\|_{H}^{2} d t\right)^{\frac{1}{2}} .
$$

By $B_{C}(0, R)$ we denote the open ball of radius $R$ centered at 0 in $C(I, H)$. The symbol $\langle\cdot, \cdot\rangle_{L^{2}}$ denotes the inner product in $L^{2}(I, H)$.

Consider the space of all absolutely continuous functions $u: I \rightarrow H$ whose generalized derivatives belong to $L^{2}(I, H)$. It is known (see, e.g., [2]) that this space can be identified with the Sobolev space $W^{1,2}(I, H)$ endowed with the norm

$$
\|u\|_{W}=\left(\|u\|_{2}^{2}+\left\|u^{\prime}\right\|_{2}^{2}\right)^{1 / 2}
$$

The embedding $W^{1,2}(I, H) \hookrightarrow C(I, H)$ is continuous, and for every $n \geq 1$ the space $W^{1,2}\left(I, H_{n}\right)$ is compactly embedded in $C\left(I, H_{n}\right)$. The weak convergence in $W^{1,2}(I, H)\left[L^{2}(I, H)\right]$ is denoted by $x_{n} \stackrel{W}{\rightarrow} x_{0}$ [respectively, $f_{n} \stackrel{L^{2}}{\rightarrow} f_{0}$ ].

By $W_{T}^{1,2}(I, H)$ we denote the subspace of all functions $x \in W^{1,2}(I, H)$ satisfying the boundary condition $x(0)=x(T)$.

Let $n \in \mathbb{N}$, and $\ell: W_{T}^{1,2}\left(I, H_{n}\right) \rightarrow L^{2}\left(I, H_{n}\right), \ell x=x^{\prime}$. Then $\ell$ is a linear Fredholm operator of index zero, and there exist the projections (see, e.g., [7]):

$$
C_{n}: W_{T}^{1,2}\left(I, H_{n}\right) \rightarrow W_{T}^{1,2}\left(I, H_{n}\right)
$$

and

$$
Q_{n}: L^{2}\left(I, H_{n}\right) \rightarrow L^{2}\left(I, H_{n}\right)
$$

such that $\operatorname{Im} C_{n}=\operatorname{Ker} \ell=H_{n}$ and $\operatorname{Ker} Q_{n}=\operatorname{Im} \ell$. If the operator

$$
\ell_{C_{n}}: \operatorname{dom} \ell \cap \operatorname{Ker} C_{n} \rightarrow \operatorname{Im} \ell
$$

is defined as the restriction of $\ell$ on $\operatorname{dom} \ell \cap \operatorname{Ker} C_{n}$, then $\ell_{C_{n}}$ is a linear isomorphism and we can define the operator $K_{C_{n}}: \operatorname{Im} \ell \rightarrow \operatorname{dom} \ell, K_{C_{n}}=\ell_{C_{n}}^{-1}$. Now, set Coker $\ell=L^{2}\left(I, H_{n}\right) / \operatorname{Im} \ell=H_{n}$; and let $\Pi_{n}: L^{2}\left(I, H_{n}\right) \rightarrow H_{n}$

$$
\Pi_{n} f=\frac{1}{T} \int_{0}^{T} f(s) d s
$$

and $\Lambda_{n}:$ Coker $\ell \rightarrow \operatorname{Ker} \ell$ be the identity map. Then the equation

$$
\ell x=y, y \in L^{2}\left(I, H_{n}\right)
$$

is equivalent to

$$
\left(i-C_{n}\right) x=\left(\Pi_{n}+K_{n}\right) y
$$


where $K_{n}: L^{2}\left(I, H_{n}\right) \rightarrow W_{T}^{1,2}\left(I, H_{n}\right)$ is given as $K_{n}=K_{C_{n}}\left(i-Q_{n}\right)$.

The space $L^{2}\left(I, H_{n}\right)$ can be decomposed as:

$$
L^{2}\left(I, H_{n}\right)=\mathcal{L}_{0}^{(n)} \oplus \mathcal{L}_{1}^{(n)},
$$

where $\mathcal{L}_{0}^{(n)}=H_{n}$ and $\mathcal{L}_{1}^{(n)}=\operatorname{Im} \ell$.

For every $f \in L^{2}\left(I, H_{n}\right)$ we denote its decomposition by

$$
f=f_{0}^{(n)}+f_{1}^{(n)}
$$

\section{MAIN RESUlT}

Consider the following periodic problem for the feedback control system:

$$
\left\{\begin{array}{l}
x^{\prime}(t) \in A x(t)+F(t, x(t))+B y(t) \text { for a.e. } t \in I, \\
y^{\prime}(t) \in G(t, x(t), y(t)) \text { for a.e. } t \in I \\
x(0)=x(T), y(0)=0
\end{array}\right.
$$

where $F: I \times E \rightarrow P(E)$ and $G: I \times E \times E \rightarrow K v(H)$ are given multimaps; $A \in \mathcal{L}(E)$ and $B \in \mathcal{L}(E, H) ; y_{0} \in H$.

(A) the restriction $A_{\left.\right|_{H}}$ belongs to $\mathcal{L}(H)$ and it is positively definite, i.e., there exists $a>0$ such that

$$
\langle A w, w\rangle_{H} \geq a\langle w, w\rangle_{H}
$$

for all $w \in H$ and a.e. $t \in I$;

$(F 1)$ for a.e. $t \in I$ multimap $F(t, \cdot): E \rightarrow P(E)$ is u.s.c.;

$(F 2)$ the restriction $F_{\left.\right|_{I \times H}}$ takes values in $K v(H)$ and is upper Carathéodory, i.e., for every $w \in H$ multimap $F(\cdot, w): I \rightarrow K v(H)$ is measurable and for a.e. $t \in I$ multimap $F(t, \cdot): H \rightarrow K v(H)$ is u.s.c.;

(F3) there is $\alpha>0$ such that

$$
\|F(t, w)\|_{H}=\max \left\{\|z\|_{H}: z \in F(t, w)\right\} \leq \alpha\left(1+\|w\|_{H}\right)
$$

for all $w \in H$ and a.e. $t \in I$.

$(G 1)$ for every $(w, z) \in E \times E$ multimap $G(\cdot, w, z): I \rightarrow K v(H)$ is measurable;

$(G 2)$ for every $t \in I$ multimap $G(t, \cdot, \cdot): E \times E \rightarrow K v(H)$ is u.s.c.; 
(G3) there exists $\beta>0$ such that

$$
\|G(t, w, z)\|_{H} \leq \beta\left(1+\|w\|_{E}+\|z\|_{E}\right),
$$

for all $(t, w, z) \in I \times E \times E$.

By a solution to problem (3.1) we mean a pair of functions $(x, y) \in W_{T}^{1,2}(I, H) \times$ $W^{1,2}(I, H)$ satisfying (3.1), or equivalently, by a solution to (3.1) we mean a function $x \in W_{T}^{1,2}(I, H)$ for which there exists $y \in W^{1,2}(I, H)$ such that the pair $(x, y)$ satisfies (3.1).

In the sequel, we need using the following statements.

Lemma 9 (see Theorem 5.2.5 [12]). Let $\mathcal{E}$ be a separable Banach space, $\Lambda$ a metric space, and $\mathcal{F}: I \times \mathcal{E} \times \Lambda \rightarrow K v(\mathcal{E})$ a multimap satisfying the following conditions:

$(\mathcal{F} 1)$ multimap $\mathcal{F}(\cdot, w, \lambda): I \rightarrow K v(\mathcal{E})$ has a measurable selection for every $(w, \lambda) \in$ $\mathcal{E} \times \Lambda$

$(\mathcal{F} 2)$ multimap $\mathcal{F}(t, \cdot, \cdot): \mathcal{E} \times \Lambda \rightarrow K v(\mathcal{E})$ is u.s.c. for a.e. $t \in I$;

$(\mathcal{F} 3)$ there is $k>0$ such that

$$
\|\mathcal{F}(t, w, \lambda)\|_{\mathcal{E}} \leq k\left(1+\|w\|_{\mathcal{E}}+\|\lambda\|_{\Lambda}\right)
$$

for all $(w, \lambda) \in \mathcal{E} \times \Lambda$ and for a.e. $t \in I$;

$(\mathcal{F} 4)$ there exists a function $\omega \in L_{+}^{1}[0, T]$ such that

$$
\chi(\mathcal{F}(t, \Omega, \Lambda)) \leq \omega(t) \chi(\Omega)
$$

for every nonempty bounded subset $\Omega \subset \mathcal{E}$, where $\chi$ denotes the Hausdoff measuare of noncompactness.

For each $\lambda \in \Lambda$ denote by $\Sigma_{u_{0}}^{\mathcal{F}(\cdot,, \lambda)}$ the solution set of the Cauchy problem

$$
\left\{\begin{array}{l}
u^{\prime}(t) \in \mathcal{F}(t, u(t), \lambda) \text { for a.e. } t \in I, \\
u(0)=u_{0} \in \mathcal{E} .
\end{array}\right.
$$

Then the multimap $\lambda \rightarrow \Sigma_{u_{0}}^{\mathcal{F}(\cdot, \cdot, \lambda)}$ is u.s.c.

Lemma 10 (see Theorem $70.12[8]$ ). Let $\mathcal{E}$ be a separable Banach space and $\mathcal{F}: I \times \mathcal{E} \rightarrow K v(\mathcal{E})$ be a multimap such that 
ON PERIODIC OSCILLATIONS FOR A CLASS OF ...

$(\mathcal{F} 1)$ multimap $\mathcal{F}(\cdot, w): I \rightarrow K v(\mathcal{E})$ has a measurable selection for every $w \in \mathcal{E} ;$

$(\mathcal{F} 2)$ multimap $\mathcal{F}(t, \cdot): \mathcal{E} \rightarrow K v(\mathcal{E})$ is completely u.s.c. for every $t \in I$;

$(\mathcal{F} 3)$ the set $\mathcal{F}(\Omega)$ is compact for every compact subset $\Omega \subset I \times \mathcal{E}$;

$(\mathcal{F} 4)$ there is $b>0$ such that

$$
\|\mathcal{F}(t, w)\|_{\mathcal{E}} \leq b\left(1+\|w\|_{\mathcal{E}}\right)
$$

for all $(t, w) \in I \times \mathcal{E}$.

Then the solution set of the Cauchy problem

$$
\left\{\begin{array}{l}
u^{\prime}(t) \in \mathcal{F}(t, u(t)), \text { for a.e. } t \in I, \\
u(0)=u_{0} \in \mathcal{E},
\end{array}\right.
$$

is an $R_{\delta}$-set in $C(I, \mathcal{E})$.

Now we are in position to present the main result of this paper.

Theorem 11. Let conditions $(A),(F 1)-(F 3)$ and $(G 1)-(G 3)$ hold. In addition, assume that

$$
a>\alpha+q^{2} \beta T e^{q \beta T}\|B\| .
$$

Then problem (3.1) has a solution.

Proof. For a given function $x \in C(I, H)$ consider the following multimap

$$
G_{x}: I \times E \rightarrow K v(E), G_{x}(t, z)=G(t, x(t), z) .
$$

It is easy to verify that $G_{x}$ satisfies all conditions in Lemma 10 . So we obtain that for every $x \in C(I, H)$ the set $\Psi_{x}$ of all solutions to the following problem

$$
\left\{\begin{array}{l}
y^{\prime}(t) \in G(t, y(t), x(t)) \text { for a.e. } t \in I \\
y(0)=0
\end{array}\right.
$$

is an $R_{\delta}$-set in $C(I, E)$.

Moreover, for a chosen number $r>0$ let $\Lambda=B_{C}(0, r)$, and consider the multimap

$$
\Pi: I \times E \times \Lambda \rightarrow K v(E), \Pi(t, w, x)=G(t, w, x(t)) .
$$


It is clear that multimap $\Pi$ satisfies conditions $(\mathcal{F} 1)-(\mathcal{F} 3)$ in Lemma 9 and for every bounded subset $\Omega \subset E$ the set $\Pi(t, \Omega, \Lambda)$ is bounded in $H$, and hence, it is a relatively compact subset in $E$. Therefore, $\Pi$ satisfies all conditions in Lemma 9. By virtue of Lemma 9 the multimap $x \rightarrow \Psi_{x}$ is u.s.c. at all points $x \in \Lambda$. Since we can choose arbitrarily $r>0$, so if we define the multimap $\Psi: C(I, H) \rightarrow K(C(I, E)), \Psi(x)=\Psi_{x}$, then it is upper semicontinuous, too.

Now define the following maps and multimaps

$$
\begin{gathered}
\widetilde{\Psi}: C(I, H) \rightarrow K(C(I, H) \times C(I, E)), \widetilde{\Psi}(x)=\{x\} \times \Psi(x), \\
B^{*}: C(I, E) \rightarrow L^{2}(I, H),\left(B^{*} y\right)(t)=B y(t), \\
\widetilde{B}: C(I, H) \times C(I, E) \rightarrow C(I, H) \times L^{2}(I, H), \widetilde{B}(x, y)=\{x\} \times\left\{B^{*} y\right\}, \\
\widetilde{A}: C(I, H) \rightarrow L^{2}(I, H),(\widetilde{A} x)(t)=A x(t), \\
\widetilde{\mathcal{P}}_{F}: C(I, H) \times L^{2}(I, H) \rightarrow C v\left(L^{2}(I, H) \times L^{2}(I, H)\right), \\
\widetilde{\mathcal{P}}_{F}(x, y)=\left\{\widetilde{A} x+\mathcal{P}_{F}(x)\right\} \times\{y\},
\end{gathered}
$$

and $\sigma: L^{2}(I, H) \times L^{2}(I, H) \rightarrow L^{2}(I, H)$,

$$
\sigma(x, y)=x+y
$$

Then problem (3.1) can be written in the form

$$
\ell x \in \sigma \circ \widetilde{\mathcal{P}}_{F} \circ \widetilde{B} \circ \widetilde{\Psi}(x)
$$

where $\ell: W_{T}^{1,2}(I, H) \rightarrow L^{2}(I, H)$ is the differentiation operator.

Let us show that the solutions of (3.2) are a priori bounded. In fact, assume that $x_{*} \in W_{T}^{1,2}(I, H)$ is a solution to (3.2). Then there exist $y_{*} \in \Psi\left(x_{*}\right)$ and $f_{*} \in \mathcal{P}_{F}\left(x_{*}\right)$ such that

$$
x_{*}^{\prime}(t)=A x_{*}(t)+f_{*}(t)+B y_{*}(t) \text { for a.e. } t \in I \text {. }
$$

Therefore,

$$
\int_{0}^{T}\left\langle A x_{*}(t)+f(t)+B y(t), x_{*}(t)\right\rangle_{H} d t=\int_{0}^{T}\left\langle x_{*}^{\prime}(t), x_{*}(t)\right\rangle_{H} d t=0 .
$$

On the other hand, for every $x \in W_{T}^{1,2}(I, H)$ and for all $y \in \Psi(x), f \in \mathcal{P}_{F}(x)$ the following estimation holds: 
ON PERIODIC OSCILLATIONS FOR A CLASS OF ...

$$
\begin{aligned}
& \int_{0}^{T}\langle A x(t)+f(t)+B y(t), x(t)\rangle_{H} d t \\
& \geq a\|x\|_{2}^{2}-\int_{0}^{T}\|x(t)\|_{H}\|f(t)\|_{H} d t-\int_{0}^{T}\|x(t)\|_{H}\|B y(t)\|_{H} d t \\
& \geq a\|x\|_{2}^{2}-\alpha \int_{0}^{T}\|x(t)\|_{H}\left(1+\|x(t)\|_{H}\right) d t-\int_{0}^{T}\|B\|\|x(t)\|_{H}\|y(t)\|_{E} d t \\
& \geq(a-\alpha)\|x\|_{2}^{2}-\alpha \sqrt{T}\|x\|_{2}-q\|B\| \int_{0}^{T}\|x(t)\|_{H}\|y(t)\|_{H} d t,
\end{aligned}
$$

where $q$ is the constant from (2.1).

From $y \in \Psi(x)$ it follows that there is $g \in L^{2}(I, H)$ such that

$$
g(t) \in G(t, x(t), y(t)) \text { for a.e. } t \in I
$$

and

$$
y(t)=\int_{0}^{t} g(s) d s, t \in I
$$

Hence,

$$
\begin{aligned}
\|y(t)\|_{H} & \leq \int_{0}^{t}\|g(t)\|_{H} d t \leq \beta \int_{0}^{t}\left(1+\|y(t)\|_{E}+\|x(t)\|_{E}\right) d t \\
& \leq \beta T+q \beta \sqrt{T}\|x\|_{2}+q \beta \int_{0}^{t}\|y(t)\|_{H} d t, t \in I .
\end{aligned}
$$

Applying the Gronwall Lemma (see, e.g., [10]) we obtain

$$
\|y(t)\|_{H} \leq \beta\left(T+q \sqrt{T}\|x\|_{2}\right) e^{q \beta T} \text { for all } t \in I .
$$

Consequently,

$$
\begin{aligned}
& \int_{0}^{T}\langle A x(t)+f(t)+B y(t), x(t)\rangle_{H} d t \\
& \geq\left(a-\alpha-q^{2} \beta T e^{q \beta T}\|B\|\right)\|x\|_{2}^{2}-\left(\alpha \sqrt{T}+q \beta\|B\| T \sqrt{T} e^{q \beta T}\right)\|x\|_{2}>0
\end{aligned}
$$

provided

$$
\|x\|_{2}>\frac{\alpha \sqrt{T}+q \beta\|B\| T \sqrt{T} e^{q \beta T}}{a-\alpha-q^{2} \beta T e^{q \beta T}\|B\|} .
$$

Therefore,

$$
\left\|x_{*}\right\|_{2} \leq \frac{\alpha \sqrt{T}+q \beta\|B\| T \sqrt{T} e^{q \beta T}}{a-\alpha-q^{2} \beta T e^{q \beta T}\|B\|} .
$$


By virtue of (F3) and (3.4) there is $K>0$ such that $\left\|x_{*}^{\prime}\right\|_{2} \leq K$. So, the solution set of inclusion (3.2) is a priori bounded in $W_{T}^{1,2}(I, H)$. From the continuous embedding $W_{T}^{1,2}(I, H) \hookrightarrow C(I, H)$ it follows that there exists $M>0$ such that $\left\|x_{*}\right\|_{C} \leq M$.

Now, let $R>M$. Then inclusion (3.2) has no solutions $x$ provided $\|x\|_{C} \geq R$. For each $n \in \mathbb{N}$ denote by the same symbol $\ell$ the restriction $\ell_{\left.\right|_{W_{T}^{1,2}\left(I, H_{n}\right)}}$ and consider the inclusion

$$
\ell x \in \mathbb{P}_{n} \circ \sigma \circ \widetilde{\mathcal{P}}_{F} \circ \widetilde{B} \circ \widetilde{\Psi}(x),
$$

or equivalently,

$$
x \in \Sigma_{n}(x)
$$

where $\Sigma_{n}: C\left(I, H_{n}\right) \rightarrow P\left(C\left(I, H_{n}\right)\right)$,

$$
\Sigma_{n}(x)=C_{n} x+\left(\Pi_{n}+K_{n}\right) \circ \mathbb{P}_{n} \circ \sigma \circ \widetilde{\mathcal{P}}_{F} \circ \widetilde{B} \circ \widetilde{\Psi}(x) .
$$

Let us show that $\Sigma_{n}$ is a completely u.s.c. $J^{c}$-multimap. Towards this goal, we define the following maps and multimaps

$$
\begin{gathered}
\widehat{\Psi}: C\left(I, H_{n}\right) \rightarrow K\left(C\left(I, H_{n}\right) \times C(I, H) \times C(I, E)\right), \\
\widehat{\Psi}(x)=\{x\} \times\{x\} \times\{\Psi(x)\}, \\
\widehat{B}: C\left(I, H_{n}\right) \times C(I, H) \times C(I, E) \rightarrow C\left(I, H_{n}\right) \times C(I, H) \times L^{2}(I, H), \\
\widehat{B}(x, u, v)=\{x\} \times\{u\} \times\left\{B^{*} v\right\}, \\
\widehat{H}: C\left(I, H_{n}\right) \times C(I, H) \times L^{2}(I, H) \rightarrow P\left(C\left(I, H_{n}\right) \times C\left(I, H_{n}\right)\right), \\
\widehat{H}(x, u, v)=\{x\} \times\left\{\left(\Pi_{n}+K_{n}\right) \mathbb{P}_{n} \circ \sigma \circ \widetilde{\mathcal{P}}_{F}(u, v)\right\}, \\
\widehat{\sigma}: C\left(I, H_{n}\right) \times C\left(I, H_{n}\right) \rightarrow C\left(I, H_{n}\right), \\
\widehat{\sigma}(x, y)=C_{n} x+y .
\end{gathered}
$$

It is clear that $\widehat{\Psi}$ is a $J$-multimap, $\widehat{B}$ and $\widehat{\sigma}$ are continuous maps and

$$
\Sigma_{n}(x)=\widehat{\sigma} \circ \widehat{H} \circ \widehat{B} \circ \widehat{\Psi}(x) .
$$

The multimap $\left(\Pi_{n}+K_{n}\right) \mathbb{P}_{n} \circ \sigma \circ \widetilde{\mathcal{P}}_{F}: C(I, H) \times L^{2}(I, H) \rightarrow P\left(C\left(I, H_{n}\right)\right)$ is a completely u.s.c. multimap with compact convex values, so it is a $J$-multimap. Therefore, $\Sigma_{n}$ is a completely u.s.c. $J^{c}$-multimap. 
Assume that there is $x \in \partial B_{C}^{(n)}(0, R)$ satisfying (3.5), where $B_{C}^{(n)}(0, R)=$ $B_{C}(0, R) \cap C\left(I, H_{n}\right)$. Then there exist $y \in \Psi(x)$ and $f \in \mathcal{P}_{F}(x)$ such that

$$
x^{\prime}(t)=\mathbb{P}_{n}(A x(t)+f(t)+B y(t)) \text {, for a.e. } t \in I .
$$

Since $x(t) \in H_{n}$ for all $t \in I$ we have

$$
\begin{aligned}
& \int_{0}^{T}\langle A x(t)+f(t)+B y(t), x(t)\rangle_{H} d t \\
= & \int_{0}^{T}\left\langle\mathbb{P}_{n} A x(t)+\mathbb{P}_{n} f(t)+\mathbb{P}_{n} B y(t), x(t)\right\rangle_{H} d t \\
= & \int_{0}^{T}\left\langle x^{\prime}(t), x(t)\right\rangle_{H} d t=0 .
\end{aligned}
$$

Consequently, $x$ satisfies relation (3.4), and hence, $\|x\|_{C}<R$ giving a contradiction.

So, the topological degree $\gamma_{n}=\operatorname{deg}\left(i-\Sigma_{n}, B_{C}^{(n)}(0, R)\right)$ is well defined. To evaluate this characteristic, we consider the following multimap

$$
\begin{gathered}
G_{n}: B_{C}^{(n)}(0, R) \times[0,1] \rightarrow K v\left(C\left(I, H_{n}\right)\right), \\
G_{n}(x, \eta)=C_{n} x+\left(\Pi_{n}+K_{n}\right) \circ \varphi\left(\mathbb{P}_{n} Q(x), \eta\right),
\end{gathered}
$$

where $Q: B_{C}^{(n)}(0, R) \rightarrow L^{2}(I, H)$,

$$
Q=\sigma \circ \widetilde{\mathcal{P}}_{F} \circ \widetilde{B} \circ \widetilde{\Psi},
$$

and $\varphi: L^{2}\left(I, H_{n}\right) \times[0,1] \rightarrow L^{2}\left(I, H_{n}\right)$,

$$
\varphi(h, \eta)=h_{0}+\eta h_{1}, h=h_{0}+h_{1} ; h_{0} \in \mathcal{L}_{0}^{(n)}, h_{1} \in \mathcal{L}_{1}^{(n)} .
$$

It is easy to verify that $G_{n}$ is a compact $J^{c}$-multimap. Assume that there is $\left(x_{*}, \eta_{*}\right) \in \partial B_{C}^{(n)}(0, R) \times[0,1]$ such that $x_{*} \in G_{n}\left(x_{*}, \eta_{*}\right)$. Then there exist $f_{*} \in$ $\mathcal{P}_{F}\left(x_{*}\right)$ and $y_{*} \in \Psi\left(x_{*}\right)$ such that

$$
\left\{\begin{array}{l}
x_{*}^{\prime}=\eta_{*} g_{*_{1}}^{(n)} \\
0=g_{*_{0}}^{(n)}
\end{array}\right.
$$

where $g_{*}^{(n)}(t)=P_{n} A x_{*}(t)+P_{n} f_{*}(t)+P_{n} B y_{*}(t), t \in I$.

If $\eta_{*} \neq 0$, then

$$
\int_{0}^{T}\left\langle x_{*}^{\prime}(t), x_{*}(t)\right\rangle_{H} d t=\frac{1}{\eta_{*}} \int_{0}^{T}\left\langle P_{n} A x_{*}(t)+P_{n} f_{*}(t)+P_{n} B y_{*}(t), x_{*}(t)\right\rangle_{H} d t>0
$$


giving a contradiction.

If $\eta_{*}=0$, then $x_{*}(t)=w \in H_{n}$ with $\|w\|_{H}=R$ for all $t \in I$. Since $\|w\|_{H}=R$ we have

$$
\int_{0}^{T}\left\langle P_{n} A w+P_{n} f(t)+P_{n} B y(t), w\right\rangle_{H} d t>0,
$$

for all $f \in \mathcal{P}_{F}(w)$ and all $y \in \Psi(w)$.

or equivalently,

$$
\left\langle\Pi_{n} g^{(n)}, w\right\rangle_{H_{n}}>0 \text { for all } g^{(n)} \in \mathbb{P}_{n} Q(w) .
$$

In particular, $\left\langle\Pi_{n} g_{*}^{(n)}, w\right\rangle_{H_{n}}>0$, giving the contradiction.

Hence, $G_{n}$ is a homotopy connecting the multifields $G_{n}(\cdot, 1)=\Sigma_{n}$ and $G_{n}(\cdot, 0)=C_{n}+\Pi_{n} \circ \mathbb{P}_{n} Q$. From relation (3.6) and the fact that multimap $G_{n}(\cdot, 0)$ takes values in $H_{n}$ we obtain

$$
\operatorname{deg}\left(i-\Sigma_{n}, B_{C}^{(n)}(0, R)\right)=\operatorname{deg}\left(-\Pi_{n} \mathbb{P}_{n} Q, B_{H}^{(n)}(0, R)\right)=(-1)^{n},
$$

where $B_{H}^{(n)}(0, R)=B_{C}^{(n)}(0, R) \cap H_{n}$.

Thus, for every $n \in \mathbb{N}$ there is a solution $x_{n} \in B_{C}^{(n)}(0, R)$ to $(3.5)$.

Now let us show that inclusion (3.2), and therefore problem (3.1), has a solution. Towards this goal, let us mention that the set $\left\{x_{n}\right\}_{n=1}^{\infty}$ is bounded in $W_{T}^{1,2}(I, H)$, and hence, it is weakly relatively compact in $W_{T}^{1,2}(I, H)$. W.l.o.g. assume that $x_{n} \stackrel{W}{\longrightarrow} x_{0}$. Therefore, $\ell x_{n} \stackrel{L^{2}}{\longrightarrow} \ell x_{0}$ and $x(t) \stackrel{H}{\rightarrow} x_{0}(t)$ for $t \in I$. From the compact embedding $H \hookrightarrow E$ we obtain

$$
x_{n}(t) \stackrel{E}{\rightarrow} x_{0}(t), t \in I .
$$

Let $y_{n} \in \Psi\left(x_{n}\right)$ and $f_{n} \in \mathcal{P}_{F}\left(x_{n}\right)$ be such that

$$
\ell x_{n}=\mathbb{P}_{n}\left(\widetilde{A} x_{n}+f_{n}+B^{*} y_{n}\right)
$$

The sequence $\left\{f_{n}\right\}_{n=1}^{\infty}$ is bounded in $L^{2}(I, H)$, and therefore it is weakly relatively compact. W.l.o.g. assume that

$$
f_{n} \stackrel{L^{2}}{\rightarrow} f_{0} \in L^{2}(I, H)
$$

The sequence $\left\{y_{n}\right\}_{n=1}^{\infty}$ is bounded in $W^{1,2}(I, H)$, and therefore it is weakly compact. W.l.o.g. assume that $y_{n} \stackrel{W}{\longrightarrow} y_{0}$. Therefore,

$$
y_{n}^{\prime} \stackrel{L^{2}}{\rightarrow} y_{0}^{\prime} \quad \text { and } \quad y_{n}(t) \stackrel{E}{\rightarrow} y_{0}(t), \quad \text { for } \quad t \in I .
$$


From the compact embedding $H \hookrightarrow E$ it follows that the embedding $W^{1,2}(I, H) \hookrightarrow$ $C(I, E)$ is compact. Consequently, $y_{n} \rightarrow y_{0}$ in $C(I, E)$, and therefore, $B^{*} y_{n} \rightarrow$ $B^{*} y_{0}$ in $L^{2}(I, H)$. Let us show that $\mathbb{P}_{n} f_{n} \stackrel{L^{2}}{\rightarrow} f_{0}$. Towards this goal, let us mention that since

$$
L^{2}(I, H)=\overline{\bigcup_{n=1}^{\infty} L^{2}\left(I, H_{n}\right)}
$$

for every element $g \in L^{2}(I, H)$ we have:

$$
\mathbb{P}_{n} g \stackrel{L^{2}(I, H)}{\longrightarrow} g
$$

Now for every $g \in L^{2}(I, H)$ we obtain

$$
\begin{aligned}
\left\langle\mathbb{P}_{n} f_{n}-f_{0}, g\right\rangle_{L^{2}} & =\left\langle\mathbb{P}_{n} f_{n}-\mathbb{P}_{n} f_{0}, g\right\rangle_{L^{2}}+\left\langle\mathbb{P}_{n} f_{0}-f_{0}, g\right\rangle_{L^{2}} \\
& =\left\langle f_{n}-f_{0}, \mathbb{P}_{n} g\right\rangle_{L^{2}}+\left\langle\mathbb{P}_{n} f_{0}-f_{0}, g\right\rangle_{L^{2}} \\
& =\left\langle f_{n}-f_{0}, g\right\rangle_{L^{2}}+\left\langle f_{n}-f_{0}, \mathbb{P}_{n} g-g\right\rangle_{L^{2}}+\left\langle\mathbb{P}_{n} f_{0}-f_{0}, g\right\rangle_{L^{2}} .
\end{aligned}
$$

Thus,

$$
\lim _{n \rightarrow \infty}\left\langle\mathbb{P}_{n} f_{n}-f_{0}, g\right\rangle_{L^{2}}=0
$$

or equivalently, $\mathbb{P}_{n} f_{n} \stackrel{L^{2}}{\rightarrow} f_{0}$.

From the diagram

$$
\ell x_{0} \stackrel{L^{2}}{\stackrel{1}{L}} \ell x_{n}=\mathbb{P}_{n}\left(\widetilde{A} x_{n}+f_{n}+B^{*} y_{n}\right) \stackrel{L^{2}}{\longrightarrow} \widetilde{A} x_{0}+f_{0}+B^{*} y_{0}
$$

it follows that $\ell x_{0}=\widetilde{A} x_{0}+f_{0}+B^{*} y_{0}$.

Let us show that $y_{0} \in \Psi\left(x_{0}\right)$. Notice that from $y_{n} \in \Psi\left(x_{n}\right)$ it follows that there is $\left\{g_{n}\right\}_{n=1}^{\infty} \subset L^{2}(I, H)$ such that $g_{n}(t) \in G\left(t, x_{n}(t), y_{n}(t)\right)$ for a.e. $t \in I$, and $y_{n}^{\prime}(t)=g_{n}(t)$ for a.e. $t \in I$. So, $g_{n} \stackrel{L^{2}}{\rightarrow} y_{0}^{\prime}$. By the Mazur Lemma (see, e.g., [6]) there is a sequence of convex combinations $\left\{\widehat{g}_{m}\right\}$

$$
\widehat{g}_{m}=\sum_{k=m}^{\infty} \lambda_{m k} g_{k}, \lambda_{m k} \geq 0 \text { and } \sum_{k=m}^{\infty} \lambda_{m k}=1,
$$

which converges in $L^{2}(I, H)$ to $y_{0}^{\prime}$. Applying Theorem $38([17])$ we again can assume w.l.o.g. that

$$
\widehat{g}_{m}(t) \stackrel{H}{\rightarrow} y_{0}^{\prime}(t)
$$

for a.e. $t \in I$. 
From (3.7) and (3.8) it follows that for every $t \in I$ and $\varepsilon>0$ there is $i_{0}=i_{0}(\varepsilon, t)$ such that

$$
G\left(t, x_{i}(t), y_{i}(t)\right) \subset O_{\varepsilon}^{H}\left(G\left(t, x_{0}(t), y_{0}(t)\right)\right), \text { for all } i \geq i_{0} .
$$

Then $g_{i}(t) \in O_{\varepsilon}^{H}\left(G\left(t, x_{0}(t), y_{0}(t)\right)\right)$ for all $i \geq i_{0}$, and hence, from the convexity of the set $O_{\varepsilon}^{H}\left(G\left(t, x_{0}(t), y_{0}(t)\right)\right)$ we have

$$
\hat{g}_{m}(t) \in O_{\varepsilon}^{H}\left(G\left(t, x_{0}(t), y_{0}(t)\right)\right), \text { for all } m \geq i_{0} .
$$

Thus, $y_{0}^{\prime}(t) \in G\left(t, x_{0}(t), y_{0}(t)\right)$ for a.e. $t \in I$, i.e., $y_{0} \in \Psi\left(x_{0}\right)$.

To complete the proof we need to prove that $f_{0} \in \mathcal{P}_{F}\left(x_{0}\right)$. Since $f_{n} \stackrel{L^{2}}{\rightarrow} f_{0}$, then w.l.o.g. we can assume that there is a sequence of convex combinations $\left\{\bar{f}_{m}\right\}$

$$
\bar{f}_{m}=\sum_{k=m}^{\infty} \lambda_{m k} f_{k}, \lambda_{m k} \geq 0 \text { and } \sum_{k=m}^{\infty} \lambda_{m k}=1,
$$

which converges to $f_{0}$ for a.e. $t \in I$.

From (3.7) and (F2) it follows that for a.e. $t \in I$ and for a given $\varepsilon>0$ there is an integer $i_{0}=i_{0}(\varepsilon, t)$ such that

$$
F\left(t, x_{i}(t)\right) \subset O_{\varepsilon}^{E}\left(F\left(t, x_{0}(t)\right)\right) \text { for all } i \geq i_{0} .
$$

Therefore,

$$
\bar{f}_{m}(t) \in O_{\varepsilon}^{E}\left(F\left(t, x_{0}(t)\right)\right) \text {, for all } m \geq i_{0} .
$$

Hence, $f_{0} \in \mathcal{P}_{F}\left(x_{0}\right)$.

\section{REFERENCES}

[1] R. Bader and W. Kryszewski, Fixed-point index for compositions of set-valued maps with proximally $\infty$-connected values on arbitrary ANR's, Set-Valued Anal. 2 (3) (1994), 459-480. doi:10.1007/BF01026835

[2] V. Barbu, Nonlinear Semigroups and Differential Equations in Banach Spaces, Noordhoff International Publishing, Leyden, 1976.

[3] Yu.G. Borisovich, B.D. Gel'man, A.D. Myshkis and V.V. Obukhovskii, Introduction to the Theory of Multivalued Maps and Differential inclusions, Second edition, Librokom, Moscow, 2011 (in Russian).

[4] Yu.G. Borisovich, B.D. Gelman, A.D. Myshkis and V.V. Obukhovskii, Topological methods in the theory of fixed points of multivalued mappings, (Russian) Uspekhi Mat. Nauk 35 (1980), 59-126. English translation: Russian Math. Surveys 35 (1980), 65-143. doi:10.1070/RM1980v035n01ABEH001548 
[5] K. Borsuk, Theory of Retracts. Monografie Matematyczne, 44, Państwowe Wydawnictwo Naukowe, Warsaw, 1967.

[6] I. Ekeland and R. Temam, Convex Analysis and Variation Problems, North Holland, Amsterdam, 1979.

[7] R.E. Gaines and J.L. Mawhin, Coincidence degree and nonlinear differential equations, Lecture Notes in Mathematics, no. 568, Springer-Verlag, Berlin-New York, 1977.

[8] L. Górniewicz, Topological Fixed Point Theory of Multivalued Mappings, 2nd edition. Topological Fixed Point Theory and Its Applications, 4. Springer, Dordrecht, 2006.

[9] L. Górniewicz, A. Granas and W. Kryszewski, On the homotopy method in the fixed point index theory of multi-valued mappings of compact absolute neighborhood retracts, J. Math. Anal. Appl. 161 (2) (1991), 457-473. doi:10.1016/0022-247X(91)90345-Z

[10] Ph. Hartman, Ordinary Differential Equations, Corrected reprint of the second (1982) edition [Birkhäuser, Boston, MA], Classics in Applied Mathematics, 38, Society for Industrial and Applied Mathematics (SIAM), Philadelphia, PA, 2002.

[11] D.M. Hyman, On decreasing sequences of compact absolute retracts, Fund Math. 64 (1969), 91-97.

[12] M. Kamenskii, V. Obukhovskii and P. Zecca, Condensing Multivalued Maps and Semilinear Differential Inclusions in Banach Spaces, de Gruyter Series in Nonlinear Analysis and Applications 7, Walter de Gruyter, Berlin-New York, 2001. doi:10.1515/9783110870893

[13] N.V. Loi, Method of guiding functions for differential inclusions in a Hilbert space, Differ. Uravn. 46 (10) (2010), 1433-1443 (in Russian); English tranl.: Differ. Equat. 46 (10) (2010), 1438-1447. doi:10.1134/S0012266110100071

[14] N.V. Loi, V. Obukhovskii and P. Zecca, Non-smooth guiding functions and periodic solutions of functional differential inclusions with infinite delay in Hilbert spaces, Fixed Point Theory 13 (2) (2012), 565-582.

[15] A.D. Myshkis, Generalizations of the theorem on a stationary point of a dynamical system inside a closed trajectory, (Russian) Math. Sb. 34 (1954), 525-540.

[16] V. Obukhovski, P. Zecca, N.V. Loi and S. Kornev, Method of Guiding Functions in Problems of Nonlinear Analysis, Lecture Notes in Math. 2076, Springer, Berlin, 2013. doi:10.1007/978-3-642-37070-0

[17] L. Schwartz, Cours d'Analyse 1, Second edition, Hermann, Paris, 1981. 
